\title{
A ONE-PHASE HYPERBOLIC STEFAN PROBLEM IN MULTI-DIMENSIONAL SPACE
}

\author{
DENING LI
}

\begin{abstract}
The hyperbolic heat transfer model is obtained by replacing the classical Fourier's law with the relaxation relation $\tau \vec{q}_{t}+\vec{q}=-k \nabla T$. The sufficient and necessary conditions are derived for the local existence and uniqueness of classical solutions for multi-D Stefan problem of hyperbolic heat transfer model where phase change is accompanied with delay of latent heat storage.
\end{abstract}

\section{INTRODUCTION}

Phase-change is a very common phenomenon occurring in physics and engineering, often connected with heat transfer. The classical heat transfer model is based upon Fourier's law that the heat flux vector $\vec{q}$ is proportional to the gradient of temperature $T$

$$
\vec{q}=-k \nabla T \text {. }
$$

This model has the inherent difficulty that it implies the physically unacceptable infinite propagation speed. Several different models have been proposed to modify Fourier's law to overcome this difficulty. One of the most popular choices is the relaxation constitutive relation in which, instead of Fourier's law, one assumes the following

$$
\tau \vec{q}_{t}+\vec{q}=-k \nabla T
$$

where constant $\tau$ is the delay time. The relation comes from the assumption that the heat flux responds to temperature gradient not instantaneously but over a period of time, see e.g. [14]. In a recent review of heat waves [6], there are very valuable detailed discussions of the origin and implications for the relation (1-2).

Combining the relaxation relation (1-2) and energy conservation with $c$ denoting the specific heat

$$
c T_{t}+\nabla \cdot \vec{q}=0
$$

Received by the editors March 6, 1989.

1980 Mathematics Subject Classification (1985 Revision). Primary 35L70, 80A20; Secondary $35 \mathrm{~L} 20$.

Key words and phrases. Hyperbolic equation, heat transfer, Stefan problem.

This work was partially supported by 1989 WVU Senate Grant. 
one gets the hyperbolic system for $\vec{q}$ and $T$

$$
\left\{\begin{array}{l}
\tau \vec{q}_{t}+\vec{q}+k \nabla T=0, \\
c T_{t}+\nabla \cdot \vec{q}=0 .
\end{array}\right.
$$

The hyperbolic system (1-4) can also be rewritten into one second order hyperbolic equation, i.e., telegrapher's equation

$$
\tau c T_{t t}+c T_{t}-k \Delta T=0 \text {. }
$$

In this paper, we want to study a phase-change problem (Stefan problem) for the equation (1-5) based upon the relaxation constitutive relation (1-2). The formulation of the phase-change problem for the hyperbolic heat transfer model $(1-4)$ or (1-5) depends upon the conditions imposed on the phase-change interface $S(t)$, which are determined from physical consideration. There are the classical conditions assuming continuous temperature across the phase-change interface, see $[2,15]$. A different condition was proposed in $[4,16]$, where the temperature is supposed to sustain a jump across the phase-change interface. The hyperbolic Stefan problem we are going to study in this paper is the one considered by Showalter and Walkington in [14], in which along with the continuous temperature assumption, the delay for storage of latent heat energy was assumed, as well as the heat flux delay (1-2).

Let $S_{0}$ be a smooth surface in $R^{n}$ dividing $R^{n}$ into two domains $\Omega_{0}^{+}$and $\Omega_{0}^{-}$. In each domain $\Omega_{0}^{+}, \Omega_{0}^{-}$, the initial temperature $T_{0}^{+}, T_{0}^{-}$and heat flux $\vec{q}_{0}^{+}, \vec{q}_{0}^{+}$are given. The problem is to determine the future temperature distribution $T^{+}(x, t), T^{-}(x, t)$, as well as the future position of the interface $S(t)$ which separates the domains $T^{+}(x, t) \geq 0$ and $T^{-}(x, t) \leq 0$.

Let $L>0$ be the latent heat, and $\tau^{ \pm}, c^{ \pm}, k^{ \pm}$be the delay time, specific heat, conductivity coefficient in the liquid and solid phases respectively. The 2-phase Stefan problem discussed in [14] is the following: Determine the hypersurface

$$
S(t)=\left\{x \in R^{n}: \phi(x, t)=0\right\}, \quad 0 \leq t<\infty,
$$

and the temperature distributions $T^{+}(x, t)$ and $T^{-}(x, t)$ in the domains $\Omega^{+}(t)$ $=\left\{x \in R^{n}: \phi(x, t)>0\right\}$ and $\Omega^{-}(t)=\left\{x \in R^{n}: \phi(x, t)<0\right\}, 0 \leq t<\infty$, such that

$$
\begin{gathered}
\tau^{ \pm} c^{ \pm} T_{t t}^{ \pm}+c^{ \pm} T_{t}^{ \pm}-k^{ \pm} \Delta T^{ \pm}=0, \quad \text { in } \Omega^{ \pm}(t) . \\
\left\{\begin{array}{c}
T^{+}(x, t)=T^{-}(x, t)=0, \\
\left(L+\left[\tau c T_{t}\right]\right) \phi_{t}=[k \nabla T] \cdot \nabla_{x} \phi,
\end{array} \text { on } S(t) .\right. \\
\left\{\begin{array}{l}
T^{ \pm}(x, 0)=T_{0}^{ \pm}(x), \\
T_{t}^{ \pm}(x, 0)=T_{1}^{ \pm}(x), \\
S(0)=S_{0}=\{x: \phi(x, 0)=0\} .
\end{array}\right.
\end{gathered}
$$


The first relation in the interface condition $(1-8)$ is the classical assumption that the temperature across the phase-change interface $S(t)$ is continuous and equals zero. The second relation in (1-8) is an expression that the storage of latent heat is also delayed over a period of length $\tau$ as well as the heat flux.

The corresponding 1-phase Stefan problem to be discussed in this paper can be stated as follows: Find the hypersurface $S(t)=\left\{x \in R^{n}: \phi(x, t)=0\right\}, 0 \leq$ $t<\infty$, and the temperature distributions $T(x, t)$ in $\Omega(t)=\left\{x \in R^{n}: \phi(x, t)>\right.$ $0\}, 0 \leq t<\infty$, such that

$$
\begin{gathered}
\tau c T_{t t}+c T_{t}-k \Delta T=0, \quad \text { in } \Omega(t) . \\
\left\{\begin{array}{l}
T(x, t)=0, \\
\left(L+\tau c T_{t}\right) \phi_{t}=k \nabla T \cdot \nabla_{x} \phi,
\end{array}\right. \\
\left\{\begin{array}{l}
T(x, 0)=T_{0}(x), \\
T_{t}(x, 0)=T_{1}(x), \\
S(0)=S_{0}=\{x: \phi(x, 0)=0\}
\end{array}\right.
\end{gathered}
$$

Showalter and Walkington in [14] discussed the Stefan problem similar to (1-10)-(1-12) and in more general form that there might be mushy regions where the substance in two phases coexists. However, only $H^{1}$ weak solutions were obtained for the 2-phase problem, assuming identical global signal speed independent of phase. In addition, an example was given that shows a 1-phase problem might be not solvable even for small time, if no extra conditions were imposed upon the initial data.

In this paper, we want to determine the conditions for the existence of classical smooth solutions for the 1-phase hyperbolic Stefan problem (1-10)(1-12). The discussion of the classical solution for 2-phase problem (1-7)-(1-9) requires development of the theory of two hyperbolic equations with coupled oblique derivative boundary conditions. We intend to deal with this problem elsewhere.

We will assume in the following that the above Stefan problems are nondegenerate. The 1-phase problem (1-10)-(1-12) is called nondegenerate if

$$
\nabla T_{0} \cdot \nabla \phi_{0} \neq 0 \text { on } S_{0} .
$$

The physical implication of (1-13) is that the phase-change interface in the considered problems are really moving. For noncompact $S_{0}$, the nondegenerate condition (1-13) is assumed to be satisfied uniformly on $S_{0}$.

A solution $(T, \phi)$ of $(1-10)-(1-12)$ is called a classical solution if $(T, \phi) \in$ $C^{2}\left(R^{n} \times R_{+}^{1}\right)$. Classical solutions for 2-phase problem (1-7)-(1-9) are defined similarly. It is obvious that certain compatible conditions on $S_{0}$ must be satisfied for the given data in order to have a smooth solution. For the 1-phase problem (1-10)-(1-12), the 0-order compatible condition is in fact the boundary 
condition (1-11) itself; i.e.

$$
\left\{\begin{array}{l}
T_{0}(x)=0, \\
\left(L+\tau c T_{1}\right) T_{1}-k\left|\nabla T_{0}\right|^{2}=0,
\end{array} \text { on } S_{0} .\right.
$$

The 1-order compatible condition can be obtained by comparing the two expressions for $T_{t t}$ on $S_{0}$, one from the equation (1-10) and another from the boundary condition (1-11). Take the tangential derivative on $S(t)$ of the relation $\left(L+\tau c T_{t}\right) T_{t}=k|\nabla T|^{2}$ along the direction perpendicular to $S_{0}$, we have

$$
\begin{array}{r}
\left(L+2 \tau c T_{1}\right)\left(T_{t t}+\nabla T_{1} \cdot \vec{\mu}\right)=2 k \nabla T_{0} \cdot \nabla T_{1}+k \nabla\left|T_{0}\right|^{2} \cdot \vec{\mu} \\
=2 k\left(\nabla T_{0} \cdot \nabla T_{1}+{ }^{t} \nabla T_{0}\left[\frac{\partial^{2} T_{0}}{\partial x_{i} \partial x_{j}}\right] \vec{\mu}\right),
\end{array}
$$

where $\vec{\mu}=-T_{1} \nabla T_{0}\left|\nabla T_{0}\right|^{-2}$. The equality of two expressions for $T_{t t}$ from (1-10) and (1-15) give the 1-order compatible condition. The higher order compatible conditions and the compatible conditions for the 2-phase problem (1-7)-(1-8) can be deduced similarly. We will omit the technical details and refer the reader to, e.g. [13].

Now we can state our main result concerning the Stefan problems (1-10)$(1-12)$.

Main Theorem. For the nondegenerate 1-phase Stefan problem (1.10)-(1.12), assume

(1) $T_{0}(x), T_{1}(x), \phi_{0}(x)$ are sufficiently smooth;

(2) on $S_{0}$, the compatible conditions are satisfied up to the order $s: s>$ $(n+1) / 2$;

(3) the following stability condition is satisfied

$$
\left|\nabla T_{0}\right|^{2}>T_{1}^{2} \frac{\tau c}{k}\left(1+\frac{L}{2 \tau c T_{1}+L}\right) .
$$

Then there exists a $t_{0}>0$ such that in $\left(0, t_{0}\right)$, there is a unique classical solution $(T, \phi)$ of (1-10)-(1-12), belonging to the space $H^{s+1} \times H^{s+1}\left(0, t_{0} ; \Omega(t)\right)$.

Remark. The stability condition (1-16) in the Main Theorem is a supplementary assumption, absent in [14], to guarantee the local existence of classical solution. The example in [14] shows that generally, one can not expect the one-phase problem to be well-posed globally, because of the hyperbolic wave property, nor even locally, if no extra conditions were imposed upon the initial data. And it is easy to check that the condition (1-16) is not satisfied by the example in [14] at the specified time.

From the stability condition (1-16), we can see the influence of the latent heat $L$ upon the phase-change interface. Since $T(x, t)>0$ in $\Omega(t)$, we know $T_{t}(x, t) \geq 0$ on $S(t)$. Therefore, (1-16) implies that on $S_{0},\left|T_{1} / \nabla T_{0}\right|$ must be smaller than the characteristic speed $\sqrt{k / \tau c}$ of the hyperbolic equation (1-10). 
In particular, if $L=0$, i.e., there is no latent heat storage during phase-change, the condition (1-16) simply implies that the initial speed for the movement of the interface must be smaller the the sound speed. This is a natural requirement, without which the problem could not be well-posed; see also [9]. However, if $L \neq 0$, the condition (1-16) is a stronger than the usual requirement that the phase interface moves slower than the sound speed in [9].

\section{PARTIAL hOdOGRAPH TRANSFORMATION}

In this section, we are going to perform the partial hodograph transformation of coordinates as in [11] to fix the moving phase-change interface in (1-10)(1-12). Since we are considering solutions in a short time interval, keeping in mind the localization technique, we may assume, without loss of generality, that

$$
\phi_{0}(x)=x^{n}-\psi\left(x^{\prime}\right)
$$

where $x^{\prime}=\left(x^{1}, \ldots, x^{n-1}\right), \psi(0)=0, \nabla \psi(0)=0$ and for some small $R>0$, $\psi\left(x^{\prime}\right)=$ const. in $\left|x^{\prime}\right|<R$. From the nondegeneracy assumption (1-13), we may take $T$ as one of the new coordinates and introduce the following partial hodograph transformation of coordinates $(t, x) \mapsto\left(y^{0}, y\right)$

$$
\left\{\begin{array}{l}
y^{0}=t, \\
y^{j}=x^{j}, j=1, \ldots, n-1, \\
y^{n}=T(x, t) .
\end{array}\right.
$$

With $y=\left(y^{1}, \ldots, y^{n}\right)$, the inverse transform of $(2-2)$ is

$$
\left\{\begin{array}{l}
t=y^{0}, x^{j}=y^{j}, j=1, \ldots, n-1, \\
x^{n}=u\left(y^{0}, y\right) .
\end{array}\right.
$$

In this new coordinate, it is obvious that the phase-change interface $S(t)$ is the coordinate plane $y^{n}=0$ and the domain $\Omega(t)$ is the halfspace $\left\{y: y^{n}>0\right\}$.

Now, we are going to rewrite the 1-phase problem (1-10)-(1-12). Denote

$$
p_{j}=\frac{\partial u}{\partial y^{j}}, \quad j=0, \ldots, n .
$$

We obtain

$$
T_{t}=-\frac{p_{0}}{p_{n}}, \quad T_{x^{j}}=-\frac{p_{j}}{p_{n}}, \quad(j=1, \ldots, n-1), \quad T_{x^{n}}=\frac{1}{p_{n}} .
$$

Therefore,

$$
\left\{\begin{aligned}
\partial_{t} & =\partial_{y^{0}}-\frac{p_{0}}{p_{n}} \partial_{y^{n}}, \\
\partial_{x^{j}} & =\partial_{y^{j}}-\frac{p_{j}}{p_{n}} \partial_{y^{n}}, \quad j=1, \ldots, n-1 \\
\partial_{x^{n}} & =\frac{1}{p_{n}} \partial_{y^{n}}
\end{aligned}\right.
$$


and

$$
\left\{\begin{aligned}
T_{t t} & =-\frac{1}{p_{n}} u_{y^{0} y^{0}}+2 \frac{p_{0}}{p_{n}^{2}} u_{y^{0} y^{n}}-\frac{p_{0}^{2}}{p_{n}^{3}} u_{y^{n} y^{n}}, \\
T_{x^{j} x^{j}} & =-\frac{1}{p_{n}} u_{y^{j} y^{j}}+2 \frac{p_{j}}{p_{n}^{2}} u_{y^{j} y^{n}}-\frac{p_{j}^{2}}{p_{n}^{3}} u_{y^{n} y^{n}}, \quad j=1, \ldots, n-1, \\
T_{x^{n} x^{n}} & =-\frac{1}{p_{n}^{3}} u_{y^{n} y^{n}} .
\end{aligned}\right.
$$

Consequently, if we define the symmetric matrix $H$,

$$
H=\left(h^{i j}\right)=\left(\begin{array}{ccccc}
\frac{\tau c}{k} & 0 & \ldots & 0 & -\frac{p_{0}}{p_{n}} \frac{\tau c}{k} \\
0 & -1 & \ldots & 0 & \frac{p_{1}}{p_{n}} \\
\ldots & \ldots & \ldots & \ldots & \ldots \\
0 & 0 & \ldots & -1 & \frac{p_{n-1}}{p_{n}} \\
-\frac{p_{0}}{p_{n}} \frac{\tau c}{k} & \frac{p_{1}}{p_{n}} & \ldots & \frac{p_{n-1}}{p_{n}} & \frac{1}{p_{n}^{2}}\left(\frac{\tau c}{k} p_{0}^{2}-\sum_{1}^{n-1} p_{j}^{2}-1\right)
\end{array}\right)
$$

then the equation $(1-10)$ can be written as

$$
\sum_{i, j=0}^{n} h^{i j} \frac{\partial^{2} u}{\partial y^{i} \partial y^{j}}+\frac{c}{k} p_{0}=0 .
$$

For the free boundary condition (1-11), the first relation is implied in the new coordinates by the boundary $y_{n}=0$ itself. The second relation becomes

$$
L p_{0} p_{n}-\tau c p_{0}^{2}+k\left(\sum_{j=1}^{n-1} p_{j}^{2}+1\right)=0
$$

or in short

$$
G\left(p_{0}, p_{j}, p_{n}\right)=0
$$

The initial condition (1-12) in the new coordinates can be written as

$$
\left\{\begin{array}{c}
u(0, y)=u_{0}(y) \\
u_{y^{0}}(0, y)=u_{1}(y)
\end{array}\right.
$$

where $u_{0}(y)$ is determined by the inverse of the invertible transformation $x \mapsto$ $y=J(x)$ :

$$
\left\{\begin{array}{l}
y^{\prime}=x^{\prime} \\
y^{n}=T_{0}(x)
\end{array}\right.
$$

i.e.,

$$
\left\{\begin{array}{l}
x^{\prime}=y^{\prime} \\
x^{n}=u_{0}(y)
\end{array}\right.
$$


and $u_{1}(y)$ is determined by $(2-5)$ :

$$
u_{1}(y)=-\frac{\partial u_{0}}{\partial y^{n}} T_{1}\left(J^{-1}(y)\right) .
$$

Therefore, after the partial hodograph transformation (2-2), the free boundary problem $(1-10)-(1-12)$ for $T$ now becomes the initial boundary problem $(2-8)-(2-10)$ for $u$ with the fixed boundary $y^{n}=0$. It is obvious that the sufficiently smooth solutions of these two problems are equivalent. In the following, we need only to discuss the nonlinear problem $(2-8)-(2-10)$ and prove the existence and uniqueness of the classical solution under the assumptions of the Main Theorem.

\section{LINEARIZED PROBLEM}

We are going to prove the Main Theorem by linear iteration. First of all, we discuss the linearized problem of (2-8)-(2-10) about a given $u\left(y^{0}, y\right)$ :

$$
\sum_{i, j=0}^{n} h^{i j} v_{y^{i} y^{j}}+\frac{c}{k} v_{y^{0}}=0, \quad \text { in } y^{0}>0, y^{n}>0
$$

$$
\begin{gathered}
\left(L p_{n}-2 \tau c p_{0}\right) v_{y^{0}}+2 k \sum_{j=1}^{n-1} p_{j} v_{y^{j}}+L p_{0} v_{y^{n}}=g\left(y^{0}, y^{\prime}\right), \quad \text { on } y^{n}=0 \\
\left\{\begin{array}{l}
v(0, y)=v_{0}(y), \\
v_{y^{0}}(0, y)=v_{1}(y),
\end{array}\right.
\end{gathered}
$$

where the left-hand side of (3-2) is obtained by computing $\sum_{j=0}^{n} G_{p_{j}} v_{y^{j}}$.

Consider the general second order linear hyperbolic equation subject to a first order derivative boundary condition

$$
\sum_{i, j=0}^{n} a^{i j} v_{y^{i} y^{j}}+\sum_{j=0}^{n} a^{j} v_{y^{j}}+a v=f\left(y^{0}, y\right), \quad \text { in } y^{n}>0, y^{0}>0
$$

$$
\sum_{j=0}^{n} b^{j} v_{y^{j}}+b v=g\left(y^{0}, y^{\prime}\right), \quad \text { on } y^{n}=0
$$

$$
\left\{\begin{aligned}
v(0, y) & =v_{0}(y) \\
v_{y^{0}}(0, y) & =v_{1}(y)
\end{aligned}\right.
$$

where the matrix $\left(a^{i j}\right)$ has 1 positive and $n$ negative eigenvalues, $d y^{0}$ is a timelike direction, and all coefficients in (3-4)(3-5) are assumed to be sufficiently smooth.

The characterization of the well-posedness for (3-4)-(3-6) has already been established, see, e.g., $[3,5,11]$. The initial-boundary value problem (3-4)-(3-6) 
is called well-posed, if its solution satisfies the energy estimate

$$
\|v\|_{1, \eta, T}^{2} \leq C\left(\frac{\|f\|_{0, \eta, T}^{2}}{\eta}+\langle g\rangle_{0, \eta, T}^{2}+\left|v_{0}\right|_{1, \eta, 0}^{2}+\left|v_{1}\right|_{0, \eta, 0}^{2}\right)
$$

where, using the notation in [11],

$$
\|f\|_{s, \eta, T}^{2}=\sum_{\alpha_{0}+|\alpha|+k \leq s} \int_{0}^{T} \int_{R_{+}^{n}} e^{-2 \eta t} \eta^{2 k}\left|\frac{\partial^{\alpha_{0}+|\alpha|} f}{\partial^{\alpha_{0}} y^{0} \partial^{\alpha} y}\right|^{2} d y d y^{0}
$$

is the standard hyperbolic $\eta$-weighted norm. $\langle g\rangle_{s, \eta, T}$ and $\left|v_{j}\right|_{s, \eta, T}$ are defined similarly on the submanifolds $y^{n}=0$ and $y^{0}=T$, and

$$
\|v\|_{s, \eta, T}^{2} \equiv \max _{0 \leq t \leq T}|v|_{s, \eta, t}^{2}+\eta\|v\|_{s, \eta, T}^{2}+\langle\langle v\rangle\rangle_{s, \eta, T}^{2}
$$

with

$$
\langle\langle v\rangle\rangle_{s, \eta, T}^{2} \equiv \sum_{k=0}^{s}\left\langle\partial_{y^{n}}^{k} v\right\rangle_{s-k, \eta, T}^{2}
$$

Then, from [3, 12], we have the following

Theorem 3.1. The linear initial-boundary value problem (3-4)-(3-6) is well-posed if the following linear stability conditions are satisfied

(1) $\left|b^{n}\right| \geq \delta>0$;

(2) $\widetilde{B}=\left(\frac{a^{n n}}{b^{n}} B+N\right)$ is a forward timelike vector with respect to the matrix $\left(a^{i j}\right)$; i.e., $\widetilde{B}^{0}>0$ and $\sum_{i, j=0}^{n} \widetilde{B}^{i} a_{i j} \widetilde{B}^{j}>0$,

where $\left(a_{i j}\right)$ is the inverse of $\left(a^{i j}\right)$, and $N=-{ }^{t}\left(a^{0 n}, \ldots, a^{n n}\right)$ is the conormal vector to the exterior normal direction $-d y^{n}$.

Sketch of proof. We are giving the sketch of the proof here to show the dependence upon the coefficients in (3-4)-(3-6) of the constant $C$ in the energy estimate (3-7). For the detailed proof of the theorem, we refer the reader to [11]. It is also true that the conditions in the theorem are necessary for the constant coefficient problem if (3-7) is replaced by a more complicated energy estimate, see $[5,12]$.

The idea of the proof is to choose an appropriate timelike vector

$$
Q=\sum_{j=0}^{n} Q^{j} \partial_{y^{j}}
$$

and then to take inner product in the $\eta$-weighted space $L^{2}\left(0, T ; R_{+}^{n}\right)$ of $(3-4)$ with $Q v$

$$
\begin{gathered}
\int_{0}^{T} \int_{R_{+}^{n}} e^{-2 \eta t}\left(\sum_{i, j=0}^{n} a^{i j} v_{y^{i} y^{j}}+\sum_{j=0}^{n} a^{j} v_{y^{j}}+a v\right) Q v d y d y^{0} \\
=\int_{0}^{T} \int_{R_{+}^{n}} e^{-2 \eta t} f Q v d y d y^{0}
\end{gathered}
$$


Integrating by parts the left side to eliminate the second derivative terms, we find the resulting interior integral is bounded below by $C \eta\|v\|_{1, \eta, T}^{2}$, the boundary integral on $y^{n}=0$ is bounded below by

$$
C\langle\langle v\rangle\rangle_{1, \eta, T}^{2}-\langle g\rangle_{0, \eta, T}^{2}-\langle v\rangle_{0, \eta, T}^{2},
$$

and the integrals on $y^{0}=0$ and $y^{0}=T$ are bounded below by

$$
C_{1}|v|_{1, \eta, T}^{2}-C_{2}\left(\left|v_{0}\right|_{1, \eta, 0}^{2}+\left|v_{1}\right|_{0, \eta, 0}^{2}\right) \text {. }
$$

The right side of (3-8) is obviously bounded by

$$
\varepsilon \eta\|v\|_{1, \eta, T}^{2}+\frac{1}{\varepsilon \eta}\|f\|_{0, \eta, T}^{2} .
$$

Therefore, for $\eta \gg 1$, the energy estimate (3-7) follows readily.

In performing the integration by parts, we have introduced the derivative of the coefficients $a^{i j}$ and $Q^{j}$. From the choice of vector $Q$, we know $Q^{j}$ is a linear combination of $a^{i j}$ and $b^{j}$. So, we conclude that in order to get the 0 -order energy estimate (3-7), we must assume $a^{i j}, b^{j} \in W^{1, \infty}$. For the principal part, we need $a^{i j}, b^{j} \in L^{\infty}$, and we need $D a^{i j}, D b^{j} \in L^{\infty}$ such that each lower order term can be bounded by $L^{2}$ norms of $\left|\partial_{y} v\right|$ and $|v|$.

For the higher order energy estimate we have the following

Theorem 3.2. Assume the problem (3-4)-(3-6) is well-posed in the sense of Theorem 3.1. Then the $H^{s+1}(s>(n+1) / 2)$ solution of (3-4)-(3-6) satisfies the following energy estimate

$$
\|v\|_{s+1, \eta, T}^{2} \leq C_{s}\left(\frac{\|f\|_{s, \eta, T}^{2}}{\eta}+\langle g\rangle_{s, \eta, T}^{2}+\left|v_{0}\right|_{s+1, \eta, 0}^{2}+\left|v_{1}\right|_{s, \eta, 0}^{2}\right),
$$

where constant $C_{s}$ depends only upon the standard (non $\eta$-weighted) $H^{s}$ norms of the coefficients in (3-4)-(3-6).

Proof. By taking tangential derivatives of the problem (3-4)-(3-6) and noticing that $y^{n}=0$ is not characteristic, one can obtain the s-order energy estimate easily (the high-order normal derivatives of $v$ on $y^{0}=0$ can be estimated by employing the equation (3-4) and the trace of $f$ on $y^{0}=0$ ). As in the case of 0 -order estimate (3-7), it is obvious that for the principal part of the energy estimate, one needs $a^{i j}, b^{j} \in L^{\infty}$, just as before. For the lower order terms, because of the Banach algebra property of Sobolev space $H^{s}(s>(n+1) / 2)$, one needs $a^{i j}, a^{j}, a, b^{j}, b \in H^{s}$. Since from the imbedding theorem, $H^{s} \subset L^{\infty}$, we conclude that the constant $C_{s}$ in (3-9) depends only upon the classical non $\eta$-weighted $H^{s}$ norms of the coefficients in (3-4)-(3-6).

Remark. Accurately speaking, since we are dealing with noncompact domain $y^{0}>0, y^{n}>0$, we should use the uniform local norm $H_{u l}^{s}$ as in [7]. But the change to the $H_{u l}^{s}$ case is straightforward and we omit it here.

Now we go back to the linearized problem (3-1)-(3-3). Using the linear stability condition in Theorem 3.1, we have the following. 
Theorem 3.3. The linearized problem (3-1)-(3-3) is well-posed and its solution satisfies the energy estimate (3-7) if (1-16) is satisfied.

Proof. We have only to check that condition (1-16) for (3-1)-(3-3) is equivalent to the linear stability conditions in Theorem 3.1.

For the problem (3-1)-(3-3), we have $b_{n}=L p_{0}$ with $L$ being a positive constant, and $p_{0}=-T_{t} / T_{x^{n}}$. Since the Stefan problem is assumed to be nondegenerate, we have $T_{x^{n}}(0,0)=\nabla T_{0}(0) \neq 0$ and $T_{t}(0,0)=T_{1}(0) \neq 0$ by (1-15). Consequently, $\left|b_{n}\right| \geq \delta>0$ is always satisfied at $(0,0)$. For the condition 2 in Theorem 3.1, straightforward computation gives

$$
\widetilde{B}=\left(\frac{h^{n n}}{L p_{0}}\left(L p_{n}-2 \tau c p_{0}\right)+\frac{p_{0}}{p_{n}} \frac{\tau c}{k}, \frac{2 h^{n n} k p_{j}}{L p_{0}}-\frac{p_{j}}{p_{n}}, 0\right)^{t}
$$

with

$$
h^{n n}=\frac{1}{p_{n}^{2}}\left(\frac{\tau c}{k} p_{0}^{2}-\sum_{1}^{n-1} p_{j}^{2}-1\right)<0 .
$$

The condition (3-11) must be satisfied because it is nothing but the physical requirement that the $T=0$ interface moves slower than sound speed. Otherwise, the problem can't be well-posed. Were (3-11) violated, the solution would be nonunique, just as in the problem discussed in [15]; see also [9] for explanation.

By (2-5), we have

$$
p_{0}=-\frac{T_{t}}{T_{x^{n}}}, \quad p_{j}=-\frac{T_{x^{j}}}{T_{x^{n}}}, \quad p_{n}=\frac{1}{T_{x^{n}}} .
$$

Therefore the component $\widetilde{B}^{0}$ has the expression

$$
\widetilde{B}^{0}=\left(L T_{t}\right)^{-1}\left[\left(\frac{\tau c}{k} T_{t}^{2}-\sum_{j=1}^{n-1} T_{x^{j}}^{2}-T_{x^{n}}^{2}\right)\left(-2 \tau c T_{t}-L\right)-\frac{\tau c}{k} L T_{t}^{2}\right]
$$

and the condition $\widetilde{B}_{0}>0$ is equivalent to

$$
\left(|\nabla T|^{2}-\frac{\tau c}{k} T_{t}^{2}\right)\left(2 \tau c T_{t}+L\right)>\frac{\tau c}{k} L T_{t}^{2} .
$$

Since $T_{t}>0,(3-13)$ can be rewritten equivalently as

$$
|\nabla T|^{2}>\frac{\tau c}{k}\left(T_{t}^{2}+\frac{L T_{t}^{2}}{2 \tau c T_{t}+L}\right) .
$$

This is exactly the condition (1-16) given in the Main Theorem of $\S 1$.

We have to show that under the assumption (3-14), the condition

$$
\sum_{i, j=0}^{n} \widetilde{B}^{i} a_{i j} \widetilde{B}^{j}>0
$$

in Theorem 3.1 is also satisfied. 
From (2-7), we compute the determinant of $H$

$$
|H|=(-1)^{n}\left(-h^{00} h^{n n}+\left(h^{0 n}\right)^{2}-h^{00} \sum_{j=1}^{n-1}\left(h^{j n}\right)^{2}\right) \text {. }
$$

At $(0,0)$,

$$
h^{j n}=\frac{p_{j}}{p_{n}}=T_{x^{j}}=0, \quad j=1, \ldots, n-1 .
$$

and

$$
-h^{00} h^{n n}+\left(h^{0 n}\right)^{2}>0 .
$$

So

$$
\begin{cases}|H|>0, & \text { for } n \text { even } \\ |H|<0, & \text { for } n \text { odd }\end{cases}
$$

At $(0,0)$, we have $H^{-1}=\left(h_{i j}\right), i, j=0, \ldots, n$, with

$$
h_{00}=|H|^{-1}(-1)^{n-1} h^{n n}
$$

and

$$
\widetilde{B}=^{t}\left(-\frac{h^{n n}}{L p_{0}}\left(2 \tau c p_{0}-L p_{n}\right)+\frac{p_{0}}{p_{n}} \frac{\tau c}{k}, 0, \ldots, 0\right) .
$$

Therefore at $(0,0)$, from $(3-18)-(3-20)$ we have

$$
\sum_{i, j=0}^{n} \widetilde{B}^{i} a_{i j} \widetilde{B}^{j}=h_{00}\left(\widetilde{B}^{0}\right)^{2}>0
$$

From the continuity of the coefficients and the independence of linear stability conditions from the choice of the coordinates follows that the linear stability conditions in Theorem 3.1 are equivalent to (1-16) in the problem (3-1)-(3-3). This completes the proof of Theorem 3.3.

\section{NONLINEAR PROBLEM}

With the preparation of solution for linearized problem in $\S 3$, we are now able to prove the local existence and uniqueness of the classical solutions for the nonlinear problem $(2-8)-(2-10)$.

First of all, we state the following uniqueness theorem.

Theorem 4.1. Assume that the conditions in the Main Theorem are satisfied, and let $u\left(y^{0}, y\right) \in C^{2}\left(0, t_{0} ; R_{+}^{n+1}\right)$ be a classical solution of problem (2-8)(2-10), and the linearized problem (3-1)-(3-3) about $u\left(y^{0}, y\right)$ satisfies the linear stability condition in Theorem 3.2 in $0 \leq t \leq t_{0}$. Then, any solution $v\left(y^{0}, y\right)$ of (2-8)-(2-10) must be identical with $u\left(y^{0}, y\right)$ in $0 \leq t \leq t_{0}$.

Proof. The proof is standard. Let $v$ be a solution of $(2-8)-(2-10)$ in the interval $0 \leq t \leq t_{1}$. Denote $(2-8)$ as $L(u) u=0$, and $M(p) p \equiv \partial_{p} G(p) p$. Therefore, by 
the Taylor formula with Cauchy integral remainder, $w=u-v$ will satisfy the problem

$$
\left\{\begin{aligned}
L(u) w & =F(u, v) w, \quad \text { in } y^{n}>0, \\
M\left(\partial_{y} u\right) w & =H\left(\partial_{y} u, \partial_{y} v\right)\left(\partial_{y} w\right)^{2}, \quad \text { on } y^{n}=0 \\
w(0, y) & =0, \quad w_{y^{0}}(0, y)=0
\end{aligned}\right.
$$

where $F, H$ are operators (depending upon $u, v$ ) of order 1 and 0 respectively. Since at $y^{0}=0$, the linear stable condition (1-17) are satisfied, by the continuity of coefficients of the problem (4-1) for $w$ is well-posed in $t \in\left[0, t_{1}\right]$ with $t_{1} \ll 1$. Keeping in mind the quadratic remainder for Newtonian iteration, from Theorem 3.1, we have for all $\eta>\eta_{0}$

$$
\|w\|_{1, \eta, t_{1}}^{2} \leq C\left(\frac{\|w\|_{1, \eta, t_{1}}^{2}}{\eta}+\widetilde{C}(\eta)\langle\langle w\rangle\rangle_{1, \eta, t_{1}}^{4}\right) .
$$

with the constant $C$ independent of $\eta$ and $\widetilde{C}(\eta)$ depending on $\eta$. In (4-2), first taking $\eta$ sufficiently large and then choosing $t_{0}$ sufficiently small, we obtain

$$
\|w\|_{1, \eta, t_{1}}^{2} \leq \frac{1}{2}\left(\|w\|_{1, \eta, t_{1}}^{2}+\langle\langle w\rangle\rangle_{1, \eta, t_{1}}^{2}\right)
$$

But this is possible only when $w=0$.

Thus far, we have proved that any solution $v\left(y^{0}, y\right)$ of $(2-8)-(2-10)$ must be identical to $u\left(y^{0}, y\right)$ in some small interval $\left(0, t_{1}\right)$. We will show that we can in fact choose $t_{1}=t_{0}$. This can be achieved by contradiction argument. Otherwise, let $t_{1}<t_{0}$ be the largest possible number such that in $\left[0, t_{1}\right] v=u$. Since at $t=t_{1}$, the linearized problem about $u$ is well-posed, and at $y^{0}=t_{1}$, $u=v$, we can take $t_{1}$ as the new initial time and consider similar problem as (4-1) and show as above that $u=v$ in some interval $\left[t_{1}, t_{1}+\varepsilon\right]$. This contradicts the definition of $t_{1}$.

For the existence of classical solution in Theorem 1.1, we will employ an iteration scheme we used in $[1,8]$, which is somewhat different and technically simpler than the one in $[10,11]$. Since under the nondegeneracy assumption, the partial hodograph transformation (2-2) is invertible, we need only to prove the existence of classical solutions for the transformed problem (2-8)-(2-10).

First of all, we eliminate the initial data. From the $s$-order compatible assumption, we know that there exists a function $\tilde{u}\left(y^{0}, y\right)$ with $\tilde{u}(0, y)=u_{0}(y)$ and $\tilde{u}_{y^{0}}(0, y)=u_{1}(y)$, such that the left-hand sides of (2-8)-(2-9) would have zero traces at $y^{0}=0$ up to the order $s-1$ if $u\left(y^{0}, y\right)$ in $(2-8)-(2-9)$ were replaced by $\tilde{u}\left(y^{0}, y\right)$. Therefore, let

$$
u\left(y^{0}, y\right) \equiv \tilde{u}\left(y^{0}, y\right)+v\left(y^{0}, y\right)
$$


and substituting into (2-8)-(2-9), we obtain the following equivalent nonlinear problem for the new unknown function $v\left(y^{0}, y\right)$

$$
\sum_{i, j=0}^{n} h^{i j} \partial_{y^{i} y^{j}}^{2} v+\sum_{j=0}^{n} h^{j} \partial_{y^{j}} v=f\left(y^{0}, y\right), \quad \text { in } y^{0}>0, y^{n}>0
$$

$$
\begin{gathered}
\left(L p_{n}-2 \tau c p_{0}\right) v_{y^{0}}+2 k \sum_{j=1}^{n-1} p_{j} v_{y^{j}}+L p_{0} v_{y^{n}}=g\left(y^{0}, y^{\prime}\right), \quad \text { on } y^{n}=0 \\
v(0, y)=0, \quad v_{y^{0}}(0, y)=0
\end{gathered}
$$

where functions $f\left(y^{0}, y\right), g\left(y^{0}, y\right)$ are known functions having zero traces at $y^{0}=0$ up to the order $s-1$, and the coefficients $p_{0}, p_{j}, p_{n}, h^{i j}$ are functions of $u\left(y^{0}, y\right)=\tilde{u}\left(y^{0}, y\right)+v\left(y^{0}, y\right)$ defined as in (2-8)-(2-9), and $h^{j}, h$ are functions of $v\left(y^{0}, y\right)$, defined by (2-8)-(2-9) and (4-4)-(4-5).

Introduce the notations of operators $\mathscr{L}(v)$ and $\mathscr{B}(v)$ :

$$
\begin{gathered}
\mathscr{L}(v) \equiv \sum_{i, j=0}^{n} h^{i j}\left(v, v_{y}\right) \partial_{y^{i} y^{j}}^{2}+\sum_{j=0}^{n} h^{j}\left(v, v_{y}\right) \partial_{y^{j}}, \\
\mathscr{B}(v) \equiv\left(L p_{n}-2 \tau c p_{0}\right) \partial_{y^{0}}+2 k \sum_{j=1}^{n} p_{j} \partial_{y^{j}}+L p_{0} \partial_{y^{n}} .
\end{gathered}
$$

We may rewrite the nonlinear problem (4-4)-(4-6) briefly as following:

$$
\begin{gathered}
\mathscr{L}(v) v=f\left(y^{0}, y\right), \\
\mathscr{B}(v) v=g\left(y^{0}, y^{\prime}\right), \\
v(0, y)=0, v_{y^{0}}(0, y)=0 .
\end{gathered}
$$

In particular, we notice that for $t_{1}$ small, the linear problem

$$
\begin{cases}\mathscr{L}(0) v=f, & \mathscr{B}(0) v=g, \\ v(0, y)=0, & v_{y^{0}}(0, y)=0\end{cases}
$$

is well-posed in $\left[0, t_{1}\right]$.

Therefore, to prove the local existence of $H^{s+1}$ solutions for (2-8)-(2-10), we need only to prove the following existence theorem for (4-7)-(4-9) with homogeneous initial data:

Theorem 4.2. Under the assumption of Theorem 1.1, there exists a $t_{0}>0$ such that there is a solution $v\left(y^{0}, y\right) \in H^{s+1}\left(0, t_{0} ; R_{+}^{n}\right)$ for the nonlinear problem (4-7)-(4-9).

Proof. The existence of solution $v\left(y^{0}, y\right)$ can be established by the following iteration scheme

$$
v_{0}\left(y^{0}, y\right)=0
$$


and $v_{j+1}\left(y^{0}, y\right)$ is determined by

$$
\left\{\begin{array}{l}
\mathscr{L}\left(v_{j}\right) v_{j+1}=f\left(y^{0}, y\right), \text { in } y^{0}>0, y^{n}>0 \\
\mathscr{B}\left(v_{j}\right) v_{j+1}=g\left(y^{0}, y\right), \text { on } y^{n}=0 \\
v_{j+1}(0, y)=0, \partial_{y^{0}} v_{j+1}(0, y)=0
\end{array}\right.
$$

We want to point out here that the iteration scheme (4-11) is different from $[10,11]$ in that all the lower order terms in (4-11) are iterated as the left-hand side while leaving the right-hand side a known function. This makes possible to fix $\eta$ at the very beginning and consequently to reduce the manipulation of $\eta$-weighted norms to the easy case of equivalent standard Sobolev norms.

First we need to show that the iteration (4-11) can proceed indefinitely in some $\left[0, t_{0}\right]$. Since $(4-11)$ is well-posed when $v_{j}=0$, it will remain to be well-posed for small $v_{j}$. For fixed $\eta$, the $\eta$-weighted norms are equivalent to the standard Sobolev $H^{s}$ norms. By the fact that $v_{j}$ has zero traces at $y^{0}=0$ and the imbedding theorem, there exists $\varepsilon_{0}>0$, such that (4-11) is well-posed in $\left[0, t_{0}\right]$ for all $v_{j}$ satisfying

$$
\left\|v_{j}\right\|_{s+1, \eta, t_{0}}^{2}<\varepsilon_{0}
$$

and the solution $v_{j+1}$ of (4-11) satisfies the energy estimate

$$
\left\|v_{j+1}\right\|_{s+1, \eta, t_{0}}^{2} \leq C_{s}\left(\frac{\|f\|_{s, \eta, t_{0}}^{2}}{\eta}+\langle g\rangle_{s, \eta, t_{0}}^{2}\right)
$$

with $C_{s}$ depending only on $\varepsilon_{0}$ and independent of $v_{j}$.

Taking $t_{0}$ sufficiently small in (4-13), we can ensure the satisfaction of the following

$$
C_{s}\left(\frac{\|f\|_{s, \eta, t_{0}}^{2}}{\eta}+\langle g\rangle_{s, \eta, t_{0}}^{2}\right) \leq \varepsilon_{0} .
$$

Therefore, the iteration (4-11) gives us a bounded sequence of $\left\{v_{0}=0, v_{1}, \ldots\right.$, $\left.v_{j}, \ldots\right\}$ in the space $H^{s+1}\left(0, t_{0} ; R_{+}^{n}\right)$ satisfying (4-12) for the chosen $\varepsilon_{0}$ and $t_{0}$.

We need also to show that $\left\{v_{j}\right\}$ converges in some weaker topology. This is standard. Let $w_{j}=v_{j+1}-v_{j}$, then we have

$$
\left\{\begin{aligned}
\mathscr{L}\left(v_{j}\right) w_{j}=-\left(\mathscr{L}\left(v_{j}\right)-\mathscr{L}\left(v_{j-1}\right)\right) v_{j}, & \text { in } y^{0}>0, y^{n}>0, \\
\mathscr{B}\left(v_{j}\right) w_{j}=-\left(\mathscr{B}\left(v_{j}\right)-\mathscr{B}\left(v_{j-1}\right)\right) v_{j}, & \text { on } y^{n}=0, \\
w_{j}(0, y)=0, \quad w_{y^{0}}(0, y)=0 . &
\end{aligned}\right.
$$

As in (4-11), the above problem (4-15) for $w_{j}$ is well-posed in $\left[0, t_{0}\right]$. By the energy estimate (4-13) and applying the Taylor formula with Cauchy integral 
remainder, we have

$$
\begin{aligned}
\left\|w_{j}\right\|_{1, \eta, t_{0}}^{2} & \leq C\left(\frac{\left\|\partial_{y} w_{j-1}\right\|_{0, \eta, t_{0}}^{2}}{\eta}+\left\|\partial_{y} w_{j-1}\right\|_{0, \eta, t_{0}}^{2}\right)\left\|v_{j}\right\|_{s, \eta, t_{0}}^{2} \\
& \leq C^{\prime} \varepsilon_{0}\left\|w_{j-1}\right\|_{1, \eta, t_{0}}^{2} .
\end{aligned}
$$

Further shrinking $\varepsilon_{0}$ if necessary, then estimate (4-16) yields the convergence of $\left\{v_{j}\right\}$ in $\eta$-weighted $H^{1}$ to $v \in H^{s+1}$ and $\|v\|_{s+1, \eta, t_{0}}^{2}<\varepsilon_{0}$ by Banach-Saks theorem. This completes the proof of Theorem 4.2.

\section{REFERENCES}

1. A. T. Bui and D. Li, Double shock fronts for hyperbolic systems of conservation laws in multidimensional space, Trans. Amer. Math. Soc. 316 (1989), 233-250.

2. A. Friedman and B. Hu, The Stefan problem for a hyperbolic heat equation, IMA Preprint $348,1987$.

3. L. Gårding, Le problème de la dérivée oblique pour l'équation des ondes, C. R. Acad Sci. Paris Sér. A-B 285 (1977), 773-775.

4. J. Greenberg, A hyperbolic heat transfer problem with phase changes, IMA J. Appl. Math. 38 (1987), 1-21.

5. M. Ikawa, A mixed problem for hyperbolic equations of second order with a first order derivative boundary condition, Publ. R.I.M.S. Kyoto Univ. 5 (1969), 119-147.

6. D. D. Joseph and Luigi Preziosi, Heat waves, Rev. Modern Phys. 61 (1989), 41-73.

7. T. Kato, The Cauchy problem for quasilinear symmetric hyperbolic systems, Arch. Rational Mech. Anal. 58 (1975), 181-205.

8. D. Li, Nonlinear initial-boundary value problem and the existence of multi-dimensional shock wave for quasilinear hyperbolic-parabolic coupled systems, Chinese Ann. Math. 8B (1987), 252-280.

9. 221-231.

10. A. Majda, The existence of multi-dimensional shock fronts, Mem. Amer. Math. Soc. $\mathbf{2 7 5}$ (1983).

11. A. Majda and E. Thomann, Multi-dimensional shock fronts for second order wave equations, Comm. Partial Differential Equations 12 (1987), 777-828.

12. S. Miyatake, Mixed problem for hyperbolic equation of second order, J. Math. Kyoto Univ. 13 (1973), 435-487.

13. J. Rauch and F. Massey, Differentiability for solutions to hyperbolic initial-boundary value problems, Trans. Amer. Math. Soc. 189 (1974), 303-318.

14. R. E. Showalter and N. J. Walkington, A hyperbolic Stefan problem, Quart. Appl. Math. 45 (1987), 769-782.

15. A. Solomon, V. Alexiades, D. Wilson and J. Drake, On the formulation of a hyperbolic Stefan problem, Quart. Appl. Math. 43 (1985), 295-304.

16. A. Solomon, V. Alexiades, D. Wilson and J. Greenberg, A hyperbolic Stefan problem with discontinuous temperature, ORNL-6216, March, 1986.

Department of Mathematics, West Virginia University, Morgantown, West Virginia 26506 